Bibliotecas. Vol 39, №2, julio - diciembre, 2021. EISSN: 1659-3286

URL: http://www.revistas.una.ac.cr/index.php/bibliotecas/index

DOI: http://dx.doi.org/10.15359/rb.39-2.4

Licencia: Creative Commons (BY-NC-SA) 4.0 Internacional

\title{
Desarrollo y ejecución de un entorno virtual de aprendizaje: acercamiento de estudiantes de bibliotecología con la infoética
}

\author{
Development and Execution of a Virtual Learning Environment: An Approach of Library \\ Science Students with Infoethics
}

Recibido: 02 de febrero de 2021

Aceptado: 03 de marzo de 2021

Publicado: 09 de diciembre de 2021

\section{Resumen}

El presente artículo muestra el progreso de los procesos de fundamentación y desarrollo de un entorno virtual de aprendizaje (EVA), para efectos de generar un primer acercamiento entre la temática de la ética de la información y estudiantes de Licenciatura de la Escuela de Bibliotecología, Documentación e Información (EBDI) de la Universidad Nacional de Costa Rica. Esto requirió primariamente del abordaje de los tópicos principales de la infoética y el análisis curricular del curso titulado Ética profesional impartido entre los años 2015-2019, para posteriormente idear y generar un EVA, utilizando la plataforma Moodle, fundamentado en la investigación basada en el diseño y en la enseñanza para la compresión. De esta forma, habiéndose desarrollado las cuatro sesiones que conformaron el curso, se obtuvo como uno de los principales resultados el que el estudiantado expresara la necesidad de incorporación de la infoética en procesos investigativos desde la bibliotecología, tanto en un plano individual como desde su enseñanza en educación superior.

Palabras clave: Entornos virtuales de aprendizaje - ética de la información bibliotecología - TIC - investigación basada en el diseño - enseñanza para la compresión.

\section{Summary}

This article shows the progress of the foundation and development of a virtual learning environment (VLE), with the purpose to generate a first approach between the subject of information ethics and posgraduate students from the Library Science, Documentation and Information School (EBDI) of the National University of Costa Rica. This required primarily, the approach to the main topics of infoethics and the curricular analysis of the course entitled Professional Ethics; between the years 2015-2019, to later devise and generate an EVA using the Moodle platform, which was based on research based on design and teaching for understanding. In this way, having developed the four sessions 
that made up the course, one of the main results obtained was that the students expressed the need to incorporate infoethics in research processes from librarianship, both at an individual level and from their learning process in higher education.

Keywords: Virtual learning environments - information ethics - library science - ICT design-based research - teaching for understanding.

\section{Introducción}

El problema planteado dentro de este artículo abarca la perspectiva de dos áreas temáticas que, aunque poseen un desarrollo epistemológico cimentado, y pese a que guardan una relación evidente, no existen, a nivel investigativo, los suficientes estudios como para que llegue a darse más que una correspondencia incipiente; referida a la ética de la información (Floridi, 2005, 2006), y a la bibliotecología.

Es necesario aclarar que dicho señalamiento fue abordado con anterioridad por Hernández (2018), quien indica esa desconexión entre las áreas arriba mencionadas, siendo desarrolladas en investigaciones que trataban ambos tópicos por separado y, en menor medida, ciertos recursos documentales con alguna relación. Esa investigación, realizada por el autor citado, se enfocó en generar esos primeros acercamientos que apuntaban hacia la elaboración de un constructo teórico que fundamentara esa relación existente, debiéndose contemplar en consecuencia no solamente las dos temáticas ya mencionadas, sino que dicho nexo debía de partir del conocimiento y su gestión.

Bajo ese mismo proceso investigativo, se parte de dicho enfoque para prever el abordaje de esta carencia por medio de una aproximación de la infoética desde la enseñanza de la Bibliotecología, siendo desarrollada además con la gestión del conocimiento, dado que esta llega a englobar todas las acciones del quehacer del profesional en bibliotecología.

En ese sentido, este artículo sitúa su desarrollo dentro del cumplimiento de uno los requerimientos para optar por el grado de Máster en la Maestría en Tecnología e Innovación Educativa (MATIE), fundamentado en la generación de un entorno virtual de aprendizaje utilizando la plataforma Moodle, implementado y desarrollado para la Escuela de 
Bibliotecología, Documentación e Información (EBDI) de la Universidad Nacional (UNA), lo cual permite además el planteamiento de una tentativa necesidad de la inclusión de la ética de la información en la enseñanza bibliotecológica.

Todo este desarrollo se da basado tanto en una investigación teórica de los tópicos generadores, como en una evidencia práctica que surja del desarrollo de un taller compuesto por cuatro sesiones, en el que participan estudiantes del grado de Licenciatura para el segundo semestre del año 2020. Siendo así, se puede acotar que en Kraemer (s.f.) se llega a relacionar indirectamente en su investigación elementos de la infoética con procesos técnicos bibliotecológicos, por medio de la descripción de materiales documentales desde la catalogación y la clasificación.

Se puede acotar también, bajo esta perspectiva, a Moreno (2014), quien arguye ciertas relaciones de una ética en el sentido amplio de la palabra, con los servicios de catalogación ofrecidos por profesionales en bibliotecología. En este caso, se recalca además la relación de lo anterior con un apoyo necesario de distintas plataformas tecnológicas para poder ser más atinentes en dichos procesos técnicos.

Una aproximación más tangible de la infoética y la bibliotecología es realizada por Figueroa (2005, 2010, 2012, 2015), al enunciar la importancia de la inclusión de la infoética en la enseñanza de la bibliotecología, su contextualización dentro de la sociedad red y el intercambio de bienes comunes de información en entornos informatizados.

En el ámbito de las TIC y el contexto de enseñanza-aprendizaje se puede citar a Morado (2018), quien aborda el tema de la construcción de los entornos virtuales de aprendizaje (EVA por sus siglas) desde el enfoque del paradigma emergente y teniendo siempre en consideración la participación activa de todos los participantes del proceso, desde la perspectiva de las comunidades de práctica. 
Bajo esa línea, la misma autora ejecuta dos talleres con docentes universitarios para definir distintos tópicos que, desde un enfoque grupal, fueron definidos como los de mayor relevancia, y que deben ser tomados en cuenta para efectos de la construcción de EVA; más específicamente se podrían mencionar los elementos que componen un entorno, aspectos de principio de diseño y lineamientos de desarrollo.

Por su parte, Pinto et al. (2015) realizaron una investigación que versa sobre el desarrollo de una herramienta automatizada, que contemple la medición del criterio de calidad dentro de contextos de enseñanza y aprendizaje en los que las TIC, y más específicamente los recursos educativos electrónicos, posean un rol fundamental en el ámbito educativo.

Esto requiere, según los autores, de un sentido de evaluación bastante definido, puesto que el significado del concepto de dicho criterio no solamente es un estándar que debe de ser alcanzado, sino, además, ser periódicamente reformado en función de lo que implica estar siempre al tanto de los cambios en las necesidades de los usuarios, para así poder suplirlas a cabalidad.

Es, asimismo, referible lo realizado por Mata (en prensa), en relación con la inclusión de las TIC en procesos de capacitación relacionada con el tema de la ética, por medio de la realización de una serie de talleres para el Colegio de Profesionales en Psicología de Costa Rica. Es válido recalcar dentro de esta investigación el apartado metodológico, puesto que estructura de una forma bastante concisa los procesos de ideación y organización de las cuatro sesiones planteadas y su estrecha relación con el uso del recurso tecnológico, como un elemento de valor agregado.

Por último, en el ámbito de la educación superior, Zempoalteca et al. (2017), resaltan el hecho de que en la actualidad el estudiantado en general tiene en las TIC los medios suficientes como para cotejar, por su propia cuenta, la educación recibida en los centros educativos, más aún si esta no cumple con sus expectativas de aprendizaje. 
En ese sentido, los autores proponen la necesaria formación en el uso de las TIC y sus consecuentes competencias digitales, inicialmente enfocada en el equipo docente, puesto que este proceso es el punto de partida para generar una retroalimentación más efectiva y de doble vía, más concretamente entre profesorado y estudiantado.

\section{Aproximación teórica al conocimiento: bibliotecología y ética de la información}

El punto de partida para poder abordar la infoética debe sustentarse en el conocimiento, resultante de la apropiación del entorno como una respuesta biológica del individuo (Maturana y Varela, 2003), y siendo comunicado desde la entidad individual hacia un ámbito social, ya que la comunicación de ideas no se fundamenta en códigos, dada la recursividad del lenguaje (Mena, 2010).

Dentro de ese proceso de comunicación, y más precisamente en la gestión de materiales documentales, se puede señalar injerencia de las bibliotecas, surgidas en Oriente y que datan desde los 300 años antes de Cristo (Orera, 1995), cuyo carácter disciplinar ha ido evolucionando según los soportes materiales en los que la información es plasmada, hasta llegar a procesos de gestión del conocimiento.

Dentro del cambio tecnológico que supone mejores condiciones para procesos de gestión del conocimiento e información, y más precisamente con la aparición y consolidación de las TIC, se determina el surgimiento de la ética de la información, definida como un "campo interdisciplinario que investiga los dilemas y conflictos morales que surgen en las interacciones entre los seres humanos y la información (creación, organización, diseminación, y uso), entre la información y las comunicaciones tecnológicas (TIC), y entre los sistemas de información" (Carbo y Smith, 2008, p.1, traducción propia).

La infoética llega entonces a sustentarse gracias a su carácter macroético y ontocéntrico a partir de su enfoque medioambiental (Bustos, 2006); su orden lógico (Floridi, 2006) desde los elementos de paciente moral (como elemento con valor informacional), agente moral (quien acomete acciones de valoración moral) y nivel de abstracción (entendiéndose como el contexto 
de este accionar); además de los factores con los que posee una relación estrecha dentro de ese contexto, más concretamente el sociológico, el científico y el cultural (Floridi, 2005).

\section{a. Mediación pedagógica y entornos virtuales de aprendizaje}

La incidencia de las TIC ha permeado también el ámbito educativo, generando, desde la perspectiva de Zempoalteca et al. (2017), que las tecnologías se hayan convertido en insumos cotidianos dentro de espacios educativos; no obstante, argumentan que dicha utilidad se ve bastante reducida, pues muchas veces se hace uso de ellos bajo contextos más propios de metodologías tradicionales.

Ante ese panorama, es que la mediación pedagógica se presta para solventar esta situación, en tanto, según señalan Chaves, Chaves y Rojas (2015, p. 161), la asociación entre la mediación y los recursos tecnológicos permite generar ambientes de aprendizaje con un mayor dinamismo y creatividad desde una planificación realizada por el docente, pudiéndose promover generación de conocimiento "por medio de una gama de herramientas tecnológicas que desarrollan los estilos de aprendizaje, las inteligencias múltiples, y a las necesidades especiales de la diversidad de aprendientes".

Bajo esa misma perspectiva, Chacón (2006), citada por León (2014), hace un repaso de los orígenes de la mediación, relacionándola con la teoría del aprendizaje significativo de David Ausubel, el cognitivismo de Jerome Bruner, la teoría del construccionismo dentro del contexto histórico-sociocultural con la propuesta de desarrollo próximo de Lev Vigotski y la intervención entre el sujeto y el objeto de Jean Piaget.

Dicho esto, y bajo el contexto propio de este escrito, es que se puede definir en los entornos virtuales de aprendizaje un medio más que viable para aprovechar de mejor manera las TIC, generando comunidades de aprendizaje fundamentadas en la mediación y el paradigma emergente. 
Este enfoque desarrollado por Morado (2017) se antepone al paradigma positivista, en tanto el primero posee un enfoque complejo, integral y holístico, que busca una interacción que parte de la simplicidad hacia la complejidad.

Lo anterior puede verse intrínseco dentro de la definición de un EVA, siendo este un espacio "en el cual aprende un individuo a través de un proceso auto dirigido, utilizando una serie de recursos que soportan el aprendizaje activo, cooperativo, progresivo e independiente, facilitando la construcción de conocimientos y la adquisición de competencias personales y profesionales" (Hiraldo, 2013, p.3).

Una ejecución efectiva de esta definición tendría que verse plasmada en el logro de concretar una educación sin distancia, puesto que según Badilla (2016) un uso asertivo de las TIC colaboraría a estrechar barreras relacionadas con la afectividad, procesos cognitivos y diferencias espacio - temporales.

Por último, para poder llegar a cumplir el propósito anterior, Morado (2017) propone un seguimiento cuidadoso en el desarrollo pertinente de unos parámetros elementales, que tienen que ver con la orientación del sitio, el desarrollo de contenidos, el diseño visual, los recursos, las actividades, la comunidad y el seguimiento docente.

\section{Metodología}

Propiamente este proceso de investigación se decantó por el paradigma constructivista que desde su definición (Ramos, 2015, p. 14) versa en el interés por responder cómo se llegan a generar "los significados y su utilización mediada por las convenciones lingüísticas dentro de los diversos contextos sociales o culturales, donde los individuos otorgan significados dentro de marcos de referencia interpretativos, basados en la historicidad y en lo cultural".

Es marcada su contraposición a una cuantificación de la realidad, más propia del positivismo y pos-positivismo, en tanto que no se enfoca en el intento de la descripción de la realidad tal cual 
se presenta desde una óptica empírica, sino más bien se perfila desde una constitución epistemológica.

Este paradigma posee una relación estrecha con el enfoque elegido, siendo este el cualitativo, en tanto, según Hernández, Fernández y Baptista (2014, p. 7), adoptándolo, es viable el "desarrollar preguntas e hipótesis antes, durante o después de la recolección y el análisis de los datos. Con frecuencia, estas actividades sirven, primero, para descubrir cuáles son las preguntas de investigación más importantes; y después, para perfeccionarlas y responderlas". Ahora bien, el método elegido para fundamentar este ejercicio investigativo fue la investigación basada en el diseño (IBD por sus siglas), dado que las características que esta posee se amoldan a los requerimientos detectados inicialmente para la investigación, además de la consecución de las metas planteadas en los objetivos de esta.

Esto se debe principalmente a que, siendo incipiente la relación señalada entre la ética de la información y la bibliotecología, se presume como necesario el desarrollar, en este caso particular, resultados tanto en contextos teóricos como pragmáticos. En efecto, hacia eso apuntan de Benito y Salinas al ahondar en la IBD (2016, p.46), argumentando que "este nuevo planteamiento surge de la necesidad de aplicar los resultados de la investigación a la práctica y al desarrollo de la teoría, enfocados ambos a la resolución de problemas prácticos".

Asociado con lo anterior, es válido mencionar que este proceso también fue trazado desde la enseñanza para la comprensión (EPC por sus siglas), permitiendo que el estudiantado profundice "en la información e ir más allá de ella, buscando reconfigurar, ampliar y aplicar los conocimientos incorporados, con el fin de reconstruirlos y producir nuevos conocimientos" (Flore y Leymonié, 2007, p.2).

Estos elementos fueron enfocados al grupo de personas participantes del curso titulado bibliotecología y ética de la información, conformado inicialmente por 37 estudiantes de Licenciatura de la carrera de Bibliotecología y Documentación de la Universidad Nacional de 
Costa Rica, cuyo contexto fue que dicho grupo ya hubiese tenido relación con la temática de la ética desde la oferta curricular de la EBDI.

Para concretar esto, se dispuso de la total colaboración de la EBDI para contar con una sesión por semana durante un mes (siendo definida como actividad curricular), en el que tres personas docentes de los cursos de licenciatura titulados Implementación de sistemas integrados de información, Debates epistemológicos de la bibliotecología y Producción editorial en la era digital, cedieran el espacio completo de una de sus lecciones para poder desarrollar las sesiones.

Dichas sesiones fueron desarrolladas en la plataforma Moodle, cuya estructura permitió que inicialmente el estudiantado ingresara al EVA para tener un primer acercamiento con cada una de las temáticas planteadas (mencionadas en el apartado de resultados), abordara recursos teóricos asociados y desarrollara actividades en las que, además, se interactuara con otros participantes para hacer una primera visualización del conocimiento generado.

Cada sesión poseía título y fecha, un video del profesor explicando el contenido de la unidad, resumen de la unidad, imagen que invitara al estudiantado a un primer análisis, un apartado de preguntas generadoras, recursos de información que dieran un insumo teórico, un apartado de actividades por desarrollar dentro del foro y el enlace para la conexión al conversatorio presencial desde la virtualidad. Cada uno de esos conversatorios se realizó a la semana siguiente de las actividades hechas por el estudiantado dentro del EVA, mediante la cuenta de Zoom de la EBDI, lo que permitió un intercambio de ideas entre profesor y el estudiantado. En el Cuadro 1 se puede contextualizar de mejor forma la calendarización el aforo inicial de participación por cada sesión, además de cada una de las sesiones:

Ahora bien, dentro del apartado de técnicas, es válido acotar lo desarrollado por Orellana y Sánchez (2006), iniciando con la mención de lectura y documentación, las cuales versan sobre la apropiación que debe realizar quien investiga todo el recurso epistemológico relacionado con 
su proceso. En el caso particular, se ahondó en las temáticas de la ética de la información, su relación con la bibliotecología y todo lo concerniente a su respectivo apartado pedagógico.

\section{Cuadro 1}

Número de sesiones, calendarización, total de participantes y estudiantes que cumplieron con todas las actividades

\begin{tabular}{cccc}
\hline Sesión & Fecha & $\begin{array}{c}\text { Participantes } \\
\text { totales }\end{array}$ & $\begin{array}{c}\text { Estudiantes que completaron } \\
\text { todas las actividades }\end{array}$ \\
\hline 1 & $09-09-2020$ & 37 & 30 \\
\hline 2 & $15-09-2020$ & 37 & 23 \\
\hline 3 & $25-09-2020$ & 36 & 27 \\
\hline 4 & $30-09-2020$ & 36 & 21
\end{tabular}

Fuente: Elaboración propia del autor 2021.

En segundo lugar, se puede aludir a las técnicas de observación y participación, que como su nombre lo señala, requiere del análisis del fenómeno tanto exógena como endógenamente. Resaltan las autoras en este caso la potenciación de este acto gracias a las características propias de las TIC en entornos virtuales.

Otra de las usadas fue la de la conversación y narración, referidas en entornos virtuales a todos los recursos textuales, audiovisuales y/o hablados para generar el proceso de mediación pedagógica, y así ir conduciendo cada una de las cuatro sesiones por el tratamiento de temas medulares asociados a dichas clases.

Por último, es reseñable el registro de datos en los entornos virtuales como una forma inicial para su tratamiento, mediante la disposición de foros de participación para el desarrollo de actividades y el anexo de herramientas externas al EVA que funjan con el mismo objetivo, permitiendo tener salvaguardados elementos para su posterior análisis. 


\section{Resultados}

Antes de iniciar con el desarrollo del curso implementado dentro del EVA, es necesario mencionar el proceso de análisis curricular a los cursos de Ética profesional ofrecidos desde la EBDI en el lapso del 2015 al 2019, para así poder determinar si dentro del abordaje epistemológico de estos se contaba con el desarrollo de la infoética o, en su defecto, si otras posturas éticas tratadas fuera de esta guardaban relación con la medular del proceso investigativo propio:

\section{Cuadro 2}

Mallas curriculares del curso Ética profesional, Escuela de Bibliotecología, Documentación e Información, (UNA)

\begin{tabular}{|c|c|c|}
\hline Año & Teorías éticas & Similitudes con la infoética \\
\hline 2015 & $\begin{array}{l}\text { Kantismo, Deontologísmo y Ética } \\
\text { de la Comunicación y del diálogo }\end{array}$ & $\begin{array}{l}\text { Formación, especialización, producción, } \\
\text { actitudes y competencias, elementos éticos } \\
\text { ligados a la selección, adquisición, } \\
\text { organización, recuperación y diseminación } \\
\text { de la información. }\end{array}$ \\
\hline 2016 & $\begin{array}{l}\text { Teoría de la virtud, Consecuencial } \\
\text { ismo, Kantismo, Deontologísmo y } \\
\text { Ética de la Comunicación y del } \\
\text { diálogo }\end{array}$ & $\begin{array}{l}\text { Formación, especialización, producción, } \\
\text { actitudes y competencias, elementos éticos } \\
\text { ligados a la selección, adquisición, } \\
\text { organización, recuperación y diseminación } \\
\text { de la información. }\end{array}$ \\
\hline 2017 & $\begin{array}{l}\text { Kantismo, Deontologísmo y Ética } \\
\text { de la Comunicación y del diálogo }\end{array}$ & $\begin{array}{l}\text { Formación, especialización, producción, } \\
\text { actitudes y competencias, elementos éticos } \\
\text { ligados a la selección, adquisición, } \\
\text { organización, recuperación y diseminación } \\
\text { de la información. }\end{array}$ \\
\hline 2018 & $\begin{array}{l}\text { Ética normativa, distintas categorías } \\
\text { éticas }\end{array}$ & $\begin{array}{l}\text { El acceso a la información como un } \\
\text { Derecho Humano. }\end{array}$ \\
\hline 2019 & $\begin{array}{l}\text { Ética altruista, Ética utilitarista (John } \\
\text { Stuart Mill), Ética del deber y el } \\
\text { sacrificio, Ética evolucionista, Ética } \\
\text { vitalista (Friedrich Nietzsche, Ética } \\
\text { de los valores (Max Sheler y } \\
\text { Principios de Bioética }\end{array}$ & $\begin{array}{l}\text { Enfoque totalmente deontológico, sobre el } \\
\text { que versan nuevas realidades sociales. }\end{array}$ \\
\hline
\end{tabular}

Fuente: EBDI, 2015-2016-2017-2018-2019. 
Como se puede observar en el Cuadro 2, estos programas tienden a tratar teorías de un arquetipo más clásico e incluso mayormente enfocado en éticas dentro del contexto epistemológico y deontológico; en lugar de enfoques ontocéntricos más consecuentes con la infoética. En ese sentido, se pudo determinar desde allí la carencia de la ética de la información en la oferta curricular.

Tomando en cuenta esto, y resaltando consecuentemente la creación del EVA, es necesario señalar la estructura general de cada una de las sesiones, para poder tener una referencia más clara de los resultados obtenidos para cada uno de los cuatro apartados.

\section{Sesión 1: Gestión del conocimiento, información y bibliotecología}

- Actividad previa basada en la rutina del pensamiento Puente 3-2-1 (Ritchhart, Church y Morrison, 2014), con la que se pretende determinar en el estudiantado una primera idea en cuanto a su posición respecto a las temáticas abordadas durante el curso (Acceso a ejemplos mediante el siguiente enlace: https://tinyurl.com/y292jspi).

- Para esta tarea, se requirió de la identificación de tres palabras relacionadas con el curso, en su gran mayoría se decantaron por las del título del curso (bibliotecología e infoética), y como tercer término fluctuó entre las TIC, alfabetización informacional y/o digital, acceso abierto, gestión del conocimiento y/o información, censura y transparencia. En este sentido, las dos preguntas por desarrollar y la frase estuvieron redactadas buscando la relación entre dichos términos seleccionados.

- Fueron tratados los temas de la creación del conocimiento como una respuesta biológica, el ciclo de gestión del conocimiento y su relación con la gestión de la información, la sociedad de la información vs. la sociedad del conocimiento, el rol del profesional en bibliotecología en torno a este panorama.

- Actividad basada en la rutina de pensamiento Conectar - Ampliar - Desafiar (Ritchhart, Church y Morrison, 2014), que pretende que el estudiantado relacione lo abordado en la unidad con elementos teóricos aprehendidos en otros cursos de su etapa universitaria, e invitarlos a la profundización de dichos temas (Acceso a ejemplos mediante el siguiente enlace: https://tinyurl.com/y6mkhctu). 
- Con la respuesta a las preguntas relacionadas con lo que el estudiantado conocía, la ampliación de la teoría de la unidad y si contra argumentarían algo; fue comprobable que la base de conocimiento del grupo sobre los temas desarrollados en la unidad es bastante afianzada, ya que en su gran mayoría mencionaron que en otros cursos se trataron dichos tópicos.

En cuanto a la profundización en las temáticas se acotó en la gran mayoría que se debía generar por medio de la investigación y, consecuentemente, de momento no se argumentó en contra respecto a la teoría abordada.

- Encuentro presencial desde la virtualidad en el que se conversaron todos los temas asociados dentro de la unidad, siendo destacable el contraste entre el saber que posee el estudiantado sobre el enfoque del conocimiento desde la perspectiva social, en contraposición con su carácter biológico.

\section{Sesión 2: Alfabetización informacional y digital}

- Los elementos teóricos tratados fueron relacionados con los nativos digitales vs. inmigrantes digitales, meta-alfabetización, alfabetización digital, alfabetización informacional, brecha digital, metáforas del medio informatizado (líquido, enjambre, sociedad transparente, intoxicación informativa), creación de capacidades en el ámbito profesional y nativos digitales y el rol del profesional en bibliotecología en este contexto.

- La actividad de esta unidad consistió en que cada estudiante elaborara un mapa mental, en el que se llegaran a relacionar los elementos que, desde un punto de vista individual, fueran más preponderantes que las dos primeras sesiones del curso (Acceso a ejemplos mediante el siguiente enlace: https://tinyurl.com/yxtg9ht4).

- Con respecto a la elaboración del mapa mental, el estudiantado perfiló los términos que, desde su punto de vista, resultaron de mayor importancia o válidos de destacar; sin embargo, llegó a faltar en casi todos la conexión entre las implicaciones de la gestión del conocimiento - información, el rol de la bibliotecología en ese contexto, los procesos de alfabetización informacional - digital y la brecha digital. 
Otro elemento por destacar es que en ninguno de los recursos se dispuso de un apartado que esquematizara lo relacionado con la generación del conocimiento desde una perspectiva biológica.

- Encuentro presencial desde la virtualidad en el que se conversaron todos los temas asociados dentro de la unidad, siendo recalcable que el estudiantado no reconozca con mayor claridad que la alfabetización informacional y digital se enmarca en la gestión del conocimiento.

\section{Sesión 3: Ética de la información}

- Los temas tratados para esta sesión versaron sobre la definición de la infoética, sus factores desde lo sociológico, científico y cultural; sus tres elementos en el agente moral, paciente moral y nivel de abstracción; y sus tres dimensiones desde El como recurso, El como producto, El como objetivo.

- Como actividad se planteó el desarrollo de la rutina de pensamiento Pensar - Inquietar Explorar (Ritchhart, Church y Morrison, 2014), que pretendió generar en el estudiantado una contextualización sobre lo que sabían sobre la ética de la información desde la comparativa con los insumos teóricos de la unidad, qué dudas les generaba este acercamiento desde una primera aproximación con la bibliotecología y cómo poder solventarlas (Acceso a ejemplos mediante el siguiente enlace: https://tinyurl.com/yyphmsay).

- En este apartado se llegó a evidenciar que, en líneas generales, el estudiantado no poseía un acercamiento significativo con la infoética, llegándola a relacionar con elementos generales como la ética y la moral, sin tener el conocimiento inicial de la relacionada con la información.

No obstante, sí destacaron que, ante ese desconocimiento inicial, es necesaria una relación más directa con el tema, tanto a nivel individual como la valoración de su inclusión en la oferta curricular en bibliotecología.

- Encuentro presencial desde la virtualidad en el que se conversaron todos los temas asociados dentro de la unidad; se puede destacar que el estudiantado expresa que el 
enfoque de las éticas recibidas en su etapa universitaria dista mucho del proporcionado por la infoética.

\section{Sesión 4: Relación de la El y la bibliotecología}

- Los temas propuestos para esta unidad trataron sobre el enfoque de la infoética desde su ontología, contraposición con las éticas deontológicas, ética de la computación vs. infoética, macroética y relación de la infoética con la bibliotecología.

- La primera tarea para esta unidad consistió en la participación del estudiantado en un Padlet alojado en el EVA, en el que argumentaron cómo la infoética puede ser relacionada con la bibliotecología, evidenciando además si desde su punto de vista se requiere de la inclusión de esta dentro de la oferta curricular de su carrera (Acceso al Padlet mediante el siguiente enlace: https://padlet.com/fahv87/praqjslz8i9mhsn7).

- En este apartado, la relación elaborada por el grupo fue hecha a partir de una visión holística de la infoética, desde un nexo directo con la bibliotecología respecto a la gestión de la información y conectando con tópicos relacionados con las TIC, la relación entre biblioteca y usuarios, infoxificación, perfil del profesional en bibliotecología respecto al entorno informatizado, creación de capacidades, entre otros.

Además, fue recalcado el enfoque de la necesidad de la inclusión de la ética de la información dentro de la oferta curricular en bibliotecología.

- Como una segunda actividad, se propuso el desarrollo de la rutina del pensamiento Puente 3-2-1 (Ritchhart, Church y Morrison, 2014) para que el estudiantado pudiera determinar si con su participación en el curso su percepción y conocimiento variaron, en asociación a la misma rutina desarrollada antes de comenzar la primera sesión del curso (Acceso a ejemplos mediante el siguiente enlace: https://tinyurl.com/y6m578yn).

- En contraposición a la actividad del mismo nombre, tipificada en la sesión 1, este desarrollo tuvo un mayor grado de especificidad en cuanto a las preguntas formuladas y la frase, ya que teniendo el estudiantado un mayor conocimiento de la infoética, las cuestionantes fueron formuladas buscando respuestas más precisas acerca de la necesidad de la relación de la infoética con la bibliotecología, tanto en un desarrollo a nivel individual como desde su adopción en la oferta curricular. 
- Encuentro presencial desde la virtualidad en el que se conversaron todos los temas asociados dentro de la unidad. Se destaca, por parte del estudiantado, que una de las mayores diferencias entre éticas clásicas y la infoética radica en que esta puede ser aplicable con mayor claridad en contextos prácticos.

\section{Discusión}

Habiéndose mostrado todo el proceso que ha constituido el desarrollo de este escrito, cabe ahora el analizar los principales elementos obtenidos del apartado de resultados, teniendo presente que en este confluyen distintos elementos que apuntan hacia la búsqueda del desarrollo bibliotecológico y no únicamente desde su fundamentación epistemológica, sino también desde las posibilidades de potenciar su enseñanza y aprendizaje mediante un mejor uso de las TIC para estos efectos.

\section{a. Necesidad de inclusión de la infoética en bibliotecología}

Como se pudo detectar durante el desarrollo del curso en el EVA, la mayoría de las opiniones del grupo de estudiantes, respecto a ese primer acercamiento con la infoética, devinieron en una necesidad expresa sobre un acercamiento de esta con la bibliotecología. Más concretamente, se expresó tanto desde un esfuerzo investigativo propio, como en una sugerencia tangible sobre la posibilidad de que la ética de la información sea incluida dentro de su formación académica.

Esta postura en realidad es bastante consecuente con lo expuesto por Figueroa (2005), quien enfatiza sobre la inclusión de este corpus teórico, partiendo desde el contexto de interacción entre el cuerpo docente y la formación de profesionales, como también a través de la generación de investigación.

Al respecto, desde el punto de vista del mismo Figueroa (2005, p.4), con la generación de estas relaciones concretas se generarían en la bibliotecología resultados específicos como, por ejemplo: 
- Un juicio crítico y habilidad para reconocer y articular conflictos éticos en el campo.

- El sentido de la responsabilidad respecto a las consecuencias de las interacciones individuales o colectivas en el campo de la información.

- El diálogo intercultural sobre la base del reconocimiento de diferentes tipos de culturas y valores sociales.

- El conocimiento básico acerca de la teoría y conceptos éticos y acerca de su relevancia en el trabajo cotidiano de los especialistas en información.

De esta manera se propiciaría un enfoque holístico en el que sean tomados en cuenta, no solamente las distintas variables que genera el entorno informacional respecto a aspectos éticos conexos con ciclos de gestión de conocimiento e información, sino también, redefinir (si fuese el caso), nuestro papel como bibliotecólogos en esos procesos sinérgicos.

\section{b. Necesidad de mayor desarrollo epistemológico en bibliotecología}

Relacionado por completo con el apartado anterior, se puede también argumentar que es detectable por medio del análisis realizado en cada una de las sesiones del curso desarrollado en el EVA, un necesario fomento de mayor generación de sustento epistemológico en la profesión.

Esto se puede señalar en que, por ejemplo, el estudiantado comentaba en las dos primeras sesiones del curso que, en cierta medida, los contenidos temáticos abordados allí habían sido vistos en el transcurso de lo que llevaban de carrera; variando quizá el enfoque con que la gestión del conocimiento e información y alfabetización digital e informacional eran relacionados unos con otros.

La falta para generar una visión holística, que contemple la relación de distintos tópicos, es mencionada por Rojas (2013) al señalar que esa:

Interrelación entre la filosofía, la teoría, la metodología y la práctica es escasamente analizada y contemplada, ya que mucha de la energía se ha concentrado en resolver 
problemas prácticos sin tomar en cuenta la fundamentación epistemológica, el pensamiento crítico, la constante evolución de conceptos y los cambios sociales. (p.2)

Se podría argumentar que esto puede tener sus bases en la definición de la bibliotecología desde una perspectiva inicial más enfocada en el servicio a su comunidad usuaria. En este caso, y haciendo uso del desarrollo de conceptos de Rojas (2013), se recae más en el desarrollo de actividades dentro del paradigma de sistema, dejando de lado que la bibliotecología se desenvuelve también dentro del orden cognitivo y social.

\section{c. Entornos virtuales de aprendizaje}

Como parte de los resultados de mayor relevancia por comentar en esta sección, no hay que dejar de lado que las posibilidades en la obtención de mejores efectos en el proceso, se potenciaron gracias a las posibilidades de acompañamiento tecno-pedagógico en el contexto del EVA. Más específicamente, se debe de partir con la elección de Moodle como plataforma para su creación, ya que al ser la misma usada en la Universidad Nacional de Costa Rica para el desarrollo de algunas secciones virtuales en los cursos, los estudiantes ya tienen cierta curva de aprendizaje como para conducirse dentro del entorno eficazmente.

No obstante, aunque existe ese conocimiento sobre el uso de la plataforma, y que además a nivel individual las aportaciones del estudiantado generalmente fueron bastante buenas, se puede señalar que el sentido de interacción que pueden propiciar las TIC es un aspecto que podría ser mayormente desarrollado en la población estudiantil bajo estudio.

Por ejemplo, en varias de las sesiones del curso fueron usadas algunas rutinas del pensamiento para generar un enfoque crítico en relación con su aproximación a la infoética y a su conexión con la bibliotecología. Las aportaciones en este sentido, a un nivel individual por parte del estudiantado, fueron bastante positivas, ya que se evidenciaba que el proceso de aprehensión y relación con otros elementos temáticos del curso era tangible en sus participaciones. 
Dicha incidencia dentro de un plano individual es compartida por Rodríguez y Castillo (2019), ya que, dentro de la esquematización de su modelo para evidenciar las potencialidades de los EVA en contextos educativos, se recalcan las ventajas en el uso de rutinas de pensamiento como uno de los elementos pedagógicos principales en su investigación. A pesar de ello, se podría argumentar que ese pensamiento crítico a nivel individual no permea de la misma forma en las contribuciones que podría realizar el estudiantado en un proceso de intercambio de ideas.

Ese señalamiento es compartido por García et al. (2010), quienes afirman que, aunque las potencialidades en el uso de plataformas para la elaboración de EVA son latentes, y que su comunidad usuaria está mayormente connotada por nativos digitales, las valoraciones en el uso de elementos como los foros distan del cómo saber usarlos, en contraposición a aportaciones dentro de esos espacios de un mayor sentido de verdadera interacción.

En este caso, es necesario generar una compatibilidad entre las habilidades ya existentes que tiene el estudiantado respecto al uso de herramientas tecnológicas y el desarrollo de competencias que les permitan socializar el conocimiento generado a un nivel individual para con el resto de los estudiantes de una forma más efectiva, aprovechando precisamente las potencialidades que ofrecen las TIC para efectos educativos.

\section{Conclusiones}

Sin lugar a duda, habiéndose desarrollado el presente texto derivado del proyecto, y exponiéndose sus elementos medulares, es que se puede llegar a aseverar que la injerencia de las TIC en procesos de gestión del conocimiento y de información, trae consigo una serie de posibilidades en diversos ámbitos, que deben de ser vistos como oportunidades de mejora en vez de amenazas.

Más concretamente, uno de los primeros aspectos que deben ser acotados bajo esa línea es la aparición de la ética de la información, como una de carácter ontológico en vez de epistemológico; esto permite un análisis de valoraciones morales respecto a cualquier elemento que posea un valor informacional, siendo esto un punto clave ante el cambio paradigmático que 
suponen las TIC. Es precisamente esa respuesta ante el advenimiento tecnológico que, dadas sus características de proveer atemporalidad y asincronización a ciclos de gestión de conocimiento e información, todas las áreas del saber, cuyo objetivo principal sea su tratamiento, han tenido que reformular algunas de sus perspectivas para mantener vigencia; entre ellas claro está se encuentra la bibliotecología.

En ese sentido, es constatable que, aunque no se pueden negar acciones concretas realizadas dentro del gremio, para poder ser pertinentes con una comunidad usuaria cada vez más heterogénea en cuanto a sus necesidades informacionales, dichos esfuerzos se han visto enfocados en dar respuesta a procesos técnicos, en detrimento de un desarrollo epistemológico de la ciencia.

Dado esto es que, como parte de ese requerimiento de profundización, en dicho desarrollo se propone la posibilidad de una relación tangible entre la infoética y la bibliotecología. La necesidad de este nexo fue señalada tanto por el grupo de estudiantes que participó del curso como de la posición propia, respecto a que esa conexión debe de surgir tanto de un esfuerzo individualizado por investigar, como con la inclusión de la ética de la información en la oferta curricular de la carrera.

Este sentido de búsqueda de innovación resultaría también beneficioso no solamente con propuestas de investigación en nuevas temáticas, sino que también es factible a través del uso de las TIC como elementos de valor agregado en contextos de enseñanza - aprendizaje. Más concretamente, con la elaboración del EVA y con apoyo de recursos de la web social, se pretende que el estudiantado aborde recursos teóricos asociados con la infoética, desarrolle consecuentemente una serie de actividades en las que, paralelamente, interactúe con otros participantes para generar una visualización del conocimiento.

Se podría argumentar aquí que, parte del atractivo que mencionó el estudiantado en relación con su acercamiento a la infoética, podría radicar tanto en los recursos tecnológicos a disposición, como en el conjunto de metodologías y métodos seleccionados para el desarrollo 
y mediación dentro del EVA. De esta forma, se puede crear un contexto provisto de mayor interactividad y, por tanto, que, bajo los intereses y necesidades individuales de los estudiantes, se genere una socialización más efectiva del conocimiento.

Ante esto, y apuntando a la ya mencionada necesidad de desarrollo epistemológico en bibliotecología, se puede afirmar que esa base investigativa permita llegar a fundamentar a dicha ciencia dentro de espacios de gestión del conocimiento, requiriendo esto de una perspectiva de creación desde una dualidad biológica y social.

Por último, dicha fundamentación epistemológica no debe de dejar de lado el desarrollo a nivel de todos los requerimientos técnicos en bibliotecología; todo lo contrario, es válido el recalcar que la injerencia de las TIC exige ambos ejercicios, atendiendo en este caso tanto la teoría como la práctica profesional.

\section{Referencias bibliográficas}

Badilla, E. (2016). Educación sin distancia. Costa Rica: Universidad Castro Carazo. https://www.castrocarazo.ac.cr/web/vida-universitaria/noticias/educacion-sin-distancia de Benito, B., Salinas, J. (2016). La Investigación Basada en Diseño en Tecnología Educativa. RIITE, 0, 44-59. http://dx.doi.org/10.6018/riite/2016/260631

Bustos, E. (2006). Metáforas de la individualidad moral y fundamentos de infoética. Isegoría, 34, 47-61. http://dx.doi.org/10.3989/isegoria.2006.i34.3

Carbo, T., Smith, M. (2008). Global information ethics: Intercultural perspectives on past and future research. Journal of the American Society for Information Science and Technology, 59 (7). http://onlinelibrary.wiley.com/doi/10.1002/asi.20851/pdf

Cháves, O., Chaves, L., Rojas, D. (2015). La realidad del uso de las TIC y su mediación pedagógica para enriquecer las clases de inglés. Revista Ensayos Pedagógicos, 10(1), 159-183. http://oaji.net/articles/2020/8084-1601993094.pdf

Escuela de Bibliotecología, Documentación e Información (2015). BGE201 Ética profesional: Programa del curso. Heredia, EBDI. 
Escuela de Bibliotecología, Documentación e Información (2016). BGE201 Ética profesional: Programa del curso. Heredia, EBDI.

Escuela de Bibliotecología, Documentación e Información (2018). BGE201 Ética profesional: Programa del curso. Heredia, EBDI.

Escuela de Bibliotecología, Documentación e Información (2019). BGE201 Ética profesional: Programa del curso. Heredia, EBDI.

Figueroa, H. (2005). Ética de la información: perspectivas bibliotecológicas. http://ru.ffyl.unam.mx:8080/bitstream/10391/948/1/Hugo\%20Figueroa\%20\%20\%C3\%89tica\%20de\%20la\%20informaci\%C3\%B3n.pdf

Figueroa, H. (2010). Vivencia y convivencia en la sociedad red [Tesis de Maestría, Universidad Nacional Autónoma de México].

Figueroa, H. (2012). Los bienes comunes de información en la era digital. En E. Morales (Ed.), El conocimiento y la información como factores de integración de América Latina (pp. 1942).

http://ru.iibi.unam.mx/jspui/bitstream/IIBI UNAM/CL535/1/conocimiento informacion fa ctores 2 hugo figueroa.pdf

Figueroa, H. (2015). Los bienes comunes de información en la sociedad red: conceptuación, modelos de gestión y tendencias [Tesis Doctoral, Universidad Nacional Autónoma de México].

Flore. E., Leymonié, J. (2007). Planificaciones de aula que promueven la comprensión. En Didáctica práctica para enseñanza media y superior (pp. 1-15). Grupo Magro. https://maristas.org.mx/gestion/web/articulos/planificaciones aula promueven compren $\underline{\text { sion.pdf }}$

Floridi, L. (2005). Por una filosofía de la información. https://praxisdigital.wordpress.com/2009/12/10/por-una-filosofia-de-la-informacionluciano-floridi/

Floridi, L. (2006). Ética de la información: su naturaleza y alcance. Isegoría, 34, 19-46. http://dx.doi.org/10.3989/isegoria.2006.i34.2 
García, M., González, V., Ramos, C. (2010). Modelos de interacción en entornos virtuales de $\begin{array}{llll}\text { aprendizaje. } & \text { Tonos } & \text { digitales, } & 19,\end{array}$ http://www.tonosdigital.es/ojs/index.php/tonos/article/view/403/281

Hernández, F. (2018). Propuesta de lineamientos para el intercambio de información científica en entornos informatizados que promuevan una conducta ética en las unidades especializadas en la temática agrícola en Costa Rica [Tesis de Licenciatura, Universidad Nacional de Costa Rica]. Repositorio institucional UNA. https://repositorio.una.ac.cr/handle/11056/14896

Hernández, R., Fernández. C., Baptista, P. (2014). Metodología de la investigación. México: McGraw Hill Education.

Hiraldo, R. (2013). Uso de los entornos virtuales de aprendizaje en la educación a distancia. https://www.uned.ac.cr/academica/edutec/memoria/ponencias/hiraldo 162.pdf

Kraemer, E. (s.f.). La ética profesional y la formación de bibliotecarios en el área de los procesos técnicos. https://www.bn.gov.ar/resources/conferences/encuentrollI/ponencia-25-MKraemer.pdf

León, G. (2014). Aproximaciones a la mediación pedagógica. Calidad en la Educación Superior, 5 (1), 136-155. https://doi.org/10.22458/caes.v5i1.348

Mata, C. (en prensa). Tecnologías digitales y educación de personas adultas: una experiencia de aprendizaje para profesionales en psicología en un contexto no formal.

Maturana, H. y Varela, F. (2003). El árbol del conocimiento: las bases biológicas del entendimiento humano. Chile: Lumen.

Mena, F. (2010). Los tejidos del caos: hermenéutica bíblica desde América Latina. Costa Rica: Editorial SEBILA

Morado, F. (2017). Educación sin distancias en entornos virtuales: comunidades de aprendientes y paradigma emergente. España: Editorial Académica Española

Morado, F. (2018). Entornos virtuales de aprendizaje complejos e innovadores: Una experiencia de creación participativa desde el paradigma emergente. Revista electrónica Educare, 22 (1), 1-17. http://dx.doi.org/10.15359/ree.22-1.18

Moreno, E. (2014). A ética no serviço de catalogação: uma revisão bibliográfica. Biblios, 55, 51 59. doi: 10.5195/biblios.2014.171 
Orellana, D., Sánchez, M. (2006). Técnicas de recolección de datos en entornos virtuales más usadas en la investigación cualitativa. Revista de Investigación educativa, 24 (1), 205222. https://revistas.um.es/rie/article/view/97661

Orera, L. (1995). Evolución histórica del concepto de biblioteconomía. Revista general de $\begin{array}{lllll}\text { información } & y & \text { documentación, } & 5 & \text { (2),73-89. }\end{array}$ https://revistas.ucm.es/index.php/RGID/article/viewFile/RGID9595220073A/11295

Pinto, M., Gómez, C., Fernández. A. y Vincianne, A. (2015). Evaluareed: desarrollo de una herramienta para la evaluación de la calidad de los recursos educativos electrónicos. $\begin{array}{lllll}\text { Investigación } & \text { bibliotecológica, } & 21 & \text { 227-248. }\end{array}$ https://dialnet.unirioja.es/servlet/articulo?codigo $=6225772$

Ramos, C. (2015). Los paradigmas de la investigación científica. Av.psicol., 23(1), 9-15. http://www.unife.edu.pe/publicaciones/revistas/psicologia/2015 1/Carlos Ramos.pdf

Ritchhart, R., Church, M., Morrison (2014). Hacer visible el pensamiento: cómo promover el compromiso, la compresión y la autonomía de los estudiantes. Argentina: Editorial Paidós SAJCF

Rodríguez, B. y Castillo, C. (2019). Entornos virtuales de aprendizaje: posibilidades y retos en el ámbito universitario. Cuenca: Universidad de Castilla-La Mancha. http://hdl.handle.net/10578/22118

Rojas, N. (2013). El usuario desde el análisis histórico, filosófico, teórico y metodológico. Bbliotecas, 31 (2), $1-14$. https://www.revistas.una.ac.cr/index.php/bibliotecas/article/view/5878/5774

Zempoalteca, B., Barragán, J., González, J., Guzmán, T. (2017). Formación en TIC y competencia digital en la docencia en instituciones públicas de educación superior. Apertura, 9 (1), 80-96. http://dx.doi.org/10.32870/Ap.v9n1.922

\section{Notas de la persona autora}

Fernando Hernández Vargas. Máster en Tecnología e Innovación Educativa de la Universidad Nacional de Costa Rica. Licenciado y Bachiller en Bibliotecología y Documentación. Académico de la Escuela de Bibliotecología, Documentación e Información. Universidad Nacional, Costa Rica Correo electrónico fernando.hernandez.vargas@una.cr. 